\title{
Fractional optimal control problem for differential system with delay argument
}

\section{G. Mohamed Bahaa*}

\section{"Correspondence:}

Bahaa_gm@yahoo.com

Present address: Department of

Mathematics, Dean of Academic

Services, Taibah University,

Al-Madinah Al-Munawarah,

Saudi Arabia

Permanent address: Department of Mathematics, Faculty of Science, Beni-Suef University, Beni-Suef, Egypt

\begin{abstract}
In this paper, we apply the classical control theory to a fractional differential system in a bounded domain. The fractional optimal control problem (FOCP) for differential system with time delay is considered. The fractional time derivative is considered in a Riemann-Liouville sense. We first study the existence and the uniqueness of the solution of the fractional differential system with time delay in a Hilbert space. Then we show that the considered optimal control problem has a unique solution. The performance index of a FOCP is considered as a function of both state and control variables, and the dynamic constraints are expressed by a partial fractional differential equation. The time horizon is fixed. Finally, we impose some constraints on the boundary control. Interpreting the Euler-Lagrange first order optimality condition with an adjoint problem defined by means of a right fractional Caputo derivative, we obtain an optimality system for the optimal control. Some examples are analyzed in detail.

MSC: 46C05; 49J20; 93C20

Keywords: fractional optimal control problems; fractional differential systems; time delay; Dirichlet and Neumann conditions; existence and uniqueness of solutions; Riemann-Liouville sense; Caputo derivative
\end{abstract}

\section{Introduction}

An optimal control problem (OCP) deals with the problem of finding a control law for a given dynamic system that minimizes a performance index in terms of the state and control variables. Various optimization problems associated with the integer optimal control of differential systems with time delays were studied recently by many authors; see for instance [1-7] and the references therein). For example in [1] Bahaa found the optimality conditions for cooperative parabolic systems governed by a Schrödinger operator.

The OCP reduces to a fractional optimal control problem (FOCP) when either the performance index or the dynamic constraints or both include at least one fractional-order derivative term. The theory of fractional differential equations has received much attention over the past 20 years, since they are important in describing the natural models such as in diffusion processes, stochastic processes, finance, and hydrology. However, the number of publications on fractional optimal control problem (FOCPs) has grown; see for example [8-20] and the references therein). For example in [9], Agrawal presented a general formulation and solution scheme for fractional optimal control problem. That is, an

(c) The Author(s) 2017. This article is distributed under the terms of the Creative Commons Attribution 4.0 International License (http://creativecommons.org/licenses/by/4.0/), which permits unrestricted use, distribution, and reproduction in any medium, provided you give appropriate credit to the original author(s) and the source, provide a link to the Creative Commons license, and indicate if changes were made. 
optimal control problem in which either the performance index or the differential equations governing the dynamics of the system or both contain at least one fractional derivative term. In that paper, the fractional derivative was defined in the Riemann-Liouville sense and the formulation was obtained by means of fractional variation principle and the Lagrange multiplier technique. Following the same technique, Frederico et al. [20] obtained a Noether-like theorem for fractional optimal control problem in the sense of Caputo. Recently, Agrawal [10] presented an eigenfunction expansion approach for a class of distributed system whose dynamics is defined in the sense of Caputo. In [13], Bahaa studied the fractional optimal control problem for variational inequalities with control constraints. In [14], Bahaa studied the fractional optimal control problem for differential systems with control constraints involving second order operator and in [15] involving infinite order operator.

Additionally, if the dynamic constraints or the performance index contain delay arguments, we are faced with a delay fractional optimal control problem (DFOCP). A strong motivation for studying and investigating the solution and the properties for fractional differential equations with time delay comes from the fact that they describe efficiently anomalous diffusion on fractals (physical objects of fractional dimension, like some amorphous semiconductors or strongly porous materials, fractional random walk, etc. Other applications occur in the following fields: fluid flow, viscoelasticity, control theory of dynamical systems, diffusive transport akin to diffusion, electrical networks, probability and statistics, dynamical processes in self-similar and porous structures, electrochemistry of corrosion, optics and signal processing, rheology, etc.

However, the existence of delay in the dynamic system may cause difficulty in the control and analysis of such systems. Therefore, constructing new analytical and numerical techniques for the delay fractional dynamic systems has become a strong topic to be considered; see [21-23] and the references therein. For instance in [21] an efficient linear programming formulation is proposed for a class of fractional-order optimal control problems with delay argument. By means of the Lagrange multiplier in the calculus of variations and using the formula for fractional integration by parts, the Euler-Lagrange equations are derived in terms of a two-point fractional boundary value problem including an advance term as well as the delay argument. In [22, 23], Jarad studied the fractional variational optimal control problems with delay argument. Various fractional optimal control problems are also studied by Mophou et al. when the fractional time derivative is expressed in the Riemann-Liouville sense. For instance, we refer to the boundary optimal control [24] and the optimal control of a fractional diffusion equation with state constraints [25]. In [26] Mophou was concerned with the optimal control of the fractional diffusion equation with delay with Caputo fractional derivatives. However, the DFOCP is an issue that still needs to be investigated as proved by the poor literature on the topic.

The formulation, the analytical scheme, and the results for some FOCPs presented in this paper are attempts to fill this gap. In this paper, fractional optimal control problems for fractional dynamic systems with deviating argument are presented. The fractional time derivative is considered in the Riemann-Liouville sense. The existence and uniqueness of solutions for such equations were proved. Fractional optimal control is characterized by the adjoint problem. By using this characterization, particular properties of fractional optimal control are proved. Using the fractional integration by parts formula, we can also construct the adjoint system to our variational formulation. By a classic result of convex 
analysis we can characterize the optimal control of a system of partial differential equations and inequalities, which can be applied to concrete fractional diffusion equations.

This paper is organized as follows. In Section 1, we introduce some definitions and preliminary results. In Section 2, we formulate the fractional Dirichlet problem. In Section 3, we show that our fractional optimal control problem holds and we give the optimality system for the optimal control. In Section 4, we formulate the fractional Neumann problem. In Section 5, the minimization problem is formulated and we state some illustrative examples. In Section 6, we state our conclusion of the paper.

\section{Some basic definitions}

In this section, we state some definitions and results which will be used later.

Let $n \in N^{*}$ and $\Omega$ be a bounded open subset of $\mathbb{R}^{n}$ with a smooth boundary $\Gamma$ of class $C^{2}$. For a time $T>0$, we set $Q=\Omega \times(0, T)$ and $\Sigma=\Gamma \times(0, T)$.

The following definitions and remarks can be found in the literature (see $[8,9,24,25])$.

Definition 2.1 Let $f: R_{+} \rightarrow R$ be a continuous function on $R^{+}$and $\beta>0$. Then the expression

$$
I_{+}^{\beta} f(t)=\frac{1}{\Gamma(\beta)} \int_{0}^{t}(t-s)^{\beta-1} f(s) d s, \quad t>0,
$$

is called the Riemann-Liouville integral of order $\beta$, where $\Gamma(\cdot)$ is the Gamma function defined for any complex number $z$ by

$$
\Gamma(z)=\int_{\infty}^{0} t^{z-1} e^{-t} d t
$$

Definition 2.2 Let $f: R_{+} \rightarrow R$. The Riemann-Liouville fractional derivative of order $\beta$ of $f$ is defined by

$$
D_{+}^{\beta} f(t)=\frac{1}{\Gamma(n-\beta)} \frac{d^{n}}{d t^{n}} \int_{0}^{t}(t-s)^{n-\beta-1} f(s) d s, \quad t>0,
$$

where $\beta \in(n-1, n), n \in N$. When $\beta$ is an integer the left derivative is replaced by $D$, the ordinary differential operator.

Definition 2.3 Let $f: R_{+} \rightarrow R$. The left Caputo fractional derivative of order $\beta$ of $f$ is defined by

$$
D_{0}^{\beta} f(t)=\frac{1}{\Gamma(n-\beta)} \int_{0}^{t}(t-s)^{n-\beta-1} f^{(n)}(s) d s, \quad t>0,
$$

where $\beta \in(n-1, n), n \in N$.

The Caputo fractional derivative is a sort of regularization in the time origin for the Riemann-Liouville fractional derivative. 
Lemma 2.4 Let $T>0, u \in C^{m}([0, T]), p \in(m-1, m), m \in N$ and $v \in C^{1}([0, T])$. Then for $t \in[0, T]$ the following properties hold:

$$
\begin{aligned}
& D_{+}^{p} v(t)=\frac{d}{d t} I_{+}^{1-p} v(t), \quad m=1, \\
& D_{+}^{p} I_{+}^{p} v(t)=v(t) ; \\
& I_{+}^{p} D_{0}^{p} u(t)=u(t)-\sum_{k=0}^{m-1} \frac{t^{k}}{k !} u^{(k)}(0) ; \\
& \lim _{t \rightarrow 0^{+}} D_{0}^{p} u(t)=\lim _{t \rightarrow 0^{+}} I_{+}^{p} u(t)=0 .
\end{aligned}
$$

From now on we set

$$
D^{\beta} f(t)=\frac{1}{\Gamma(1-\beta)} \int_{t}^{T}(s-t)^{-\beta} f^{\prime}(s) d s
$$

Remark 2.5 $-D^{\beta} f(t)$ is the so-called right fractional Caputo derivative. It represent the future state of $f(t)$. For more details on the derivative we refer to $[8,9,24,25]$. Note also that, when $T=+\infty, D^{\beta} f(t)$ is the Weyl fractional integral of order $\beta$ of $f^{\prime}$.

Lemma 2.6 Let $0<\beta<1$. Then for any $\phi \in C^{\infty}(\bar{Q})$ we have $($ see $[24,25])$

$$
\begin{aligned}
\int_{0}^{T} & \int_{\Omega}\left(D_{+}^{\beta} y(x, t)+\mathcal{A}(t) y(x, t)\right) \phi(x, t) d x d t \\
= & \int_{\Omega} \phi(x, T) I_{+}^{1-\beta} y(x, T) d x-\int_{\Omega} \phi(x, 0) I_{+}^{1-\beta} y\left(x, 0^{+}\right) d x \\
& \quad+\int_{0}^{T} \int_{\partial \Omega} y \frac{\partial \phi}{\partial \nu} d \Gamma d t-\int_{0}^{T} \int_{\partial \Omega} \frac{\partial y}{\partial \nu} \phi d \Gamma d t \\
& +\int_{0}^{T} \int_{\Omega} y(x, t)\left(-D^{\beta} \phi(x, t)+\mathcal{A}^{*}(t) \phi(x, t)\right) d x d t
\end{aligned}
$$

where $\mathcal{A}(t)$ is a given operator which is defined by (3.5) below and

$$
\frac{\partial y}{\partial \nu_{\mathcal{A}(t)}}=\sum_{i, j=1}^{n} a_{i j} \frac{\partial y}{\partial x_{j}} \cos \left(n, x_{j}\right) \quad \text { on } \Gamma,
$$

$\cos \left(n, x_{j}\right)$ is the ith direction cosine of $n, n$ being the normal at $\Gamma$ exterior to $\Omega$.

We also introduce the space

$$
\mathcal{W}(0, T):=\left\{y ; y \in L^{2}\left(0, T ; H_{0}^{1}(\Omega)\right), D_{+}^{\beta} y(t) \in L^{2}\left(0, T ; H_{0}^{-1}(\Omega)\right)\right\}
$$

in which a solution of a differential systems is contained. The spaces considered in this paper are assumed to be real. 


\section{Fractional Dirichlet problem for differential equations with time lags}

Let us consider the fractional partial differential equations: suppose $\omega>0$ be given; we search $y(t) \in \mathcal{W}(0, T)$ such that

$$
\begin{aligned}
& D_{+}^{\beta} y(t)+\mathcal{A} y(t)+y(t-\omega)=f_{1}(t), \quad x \in \Omega, t \in(\omega, T), \\
& y(x, t)=g(x, t), \quad x \in \Omega, t \in(0, \omega), \\
& I_{+}^{1-\beta} y\left(x, 0^{+}\right)=y_{0}(x), \quad x \in \Omega, \\
& y(x, t)=0, \quad x \in \Gamma, t \in(0, T),
\end{aligned}
$$

with $0<\beta<1, y_{0}(x) \in H^{2}(\Omega) \cap H_{0}^{1}(\Omega)$, the function $f$ belongs to $L^{2}(Q)$. The function $g(x, t)$ belongs to $\mathcal{W}(0, \omega)$. The fractional integral $I_{+}^{1-\beta}$ and the derivative $D_{+}^{\beta}$ are understood here in the Riemann-Liouville sense, $\Omega$ is given in Section 1 and $I_{+}^{1-\beta} y\left(0^{+}\right)=\lim _{t \rightarrow 0^{+}} I_{+}^{1-\beta} y(t)$. The operator $\mathcal{A}(t) \in \mathcal{L}\left(H_{0}^{1}(\Omega), H_{0}^{-1}(\Omega)\right)$ in the state equation (3.1) is a second order selfadjoint operator given by

$$
\mathcal{A}(t) y(x, t)=-\sum_{i, j=1}^{n} \frac{\partial}{\partial x_{i}}\left(a_{i j}(x, t) \frac{\partial y(x, t)}{\partial x_{j}}\right)+a_{0}(x, t) y(x, t),
$$

where $a_{i j}(x, t), i, j=1, \ldots, n$, is a given function on $\Omega$ with the properties

$$
\begin{aligned}
& a_{0}(x, t), a_{i j}(x, t) \in L^{\infty}(\Omega) \quad \text { (with real values), } \\
& a_{0}(x, t) \geq \alpha>0, \quad \sum_{i, j=1}^{n} a_{i j}(x, t) \xi_{i} \xi_{j} \geq \alpha\left(\xi_{1}^{2}+\cdots+\xi_{n}^{2}\right), \quad \forall \xi \in R^{n},
\end{aligned}
$$

a.e. on $\Omega$.

Definition 3.1 On $H_{0}^{1}(\Omega)$ we define for each $\left.t \in\right] 0, t[$ the following bilinear form:

$$
\pi(t ; y, \phi)=(\mathcal{A}(t) y, \phi)_{L^{2}(\Omega)}, \quad y, \phi \in H_{0}^{1}(\Omega) .
$$

Then

$$
\begin{aligned}
\pi(t ; y, \phi) & =(\mathcal{A}(t) y, \phi)_{L^{2}(\Omega)} \\
& =\left(-\sum_{i, j=1}^{n} \frac{\partial}{\partial x_{i}}\left(a_{i j}(x, t) \frac{\partial y}{\partial x_{j}}\right)+a_{0}(x, t) y, \phi(x, t)\right)_{L^{2}(\Omega)} \\
& =\int_{\Omega} \sum_{i, j=1}^{n} a_{i j}(x, t) \frac{\partial}{\partial x_{i}} y(x, t) \frac{\partial}{\partial x_{j}} \phi(x, t) d x+\int_{\Omega} a_{0}(x, t) y(x, t) \phi(x, t) d x .
\end{aligned}
$$

Lemma 3.2 The bilinear form (3.7) is coercive on $H_{0}^{1}(\Omega)$, that is,

$$
\pi(t ; y, y) \geq \lambda\|y\|_{H_{0}^{\infty}\left\{a_{\alpha}, 2\right\}(\Omega)}^{2}, \quad \lambda>0
$$


Proof

$$
\begin{aligned}
\pi(t ; y, y) & =\int_{\Omega} \sum_{i, j=1}^{n} a_{i j}(x, t) \frac{\partial}{\partial x_{i}} y(x, t) \frac{\partial}{\partial x_{j}} y(x, t) d x+\int_{\Omega} a_{0}(x, t) y(x, t) y(x, t) d x \\
& =\sum_{i, j=1}^{n} a_{i j}(x, t)\left\|\frac{\partial}{\partial x_{i}} y(x, t)\right\|_{L^{2}(\Omega)}^{2}+\|y(x, t)\|_{L^{2}(\Omega)}^{2} \\
& \geq \lambda\|y\|_{H_{0}^{1}(\Omega)}^{2}, \quad \lambda>0 .
\end{aligned}
$$

Also we can assume that $\forall y, \phi \in H_{0}^{1}(\Omega)$ the bilinear form $t \rightarrow \pi(t ; y, \phi)$ is continuously differentiable in $] 0, T$ [ and the bilinear form (3.7) is symmetric,

$$
\pi(t ; y, \phi)=\pi(t ; \phi, y) \quad \forall y, \phi \in H_{0}^{1}(\Omega)
$$

The system of equations (3.1)-(3.4) defines our fractional problem with time delay. By using the Lax-Milgram lemma, we can study the existence of a unique solution of the fractional system (3.1)-(3.4).

Lemma 3.3 (see $[8,9,24,25]$ (fractional Green's formula)) Let y be the solution of system (3.1)-(3.4). Then we have, for any $\phi \in C^{\infty}(\bar{Q})$ so that $\phi(x, T)=0$ in $\Omega$ and $\phi=0$ on $\Sigma$,

$$
\begin{aligned}
\int_{0}^{T} & \int_{\Omega}\left(D_{+}^{\beta} y(x, t)+\mathcal{A}(t) y(x, t)\right) \phi(x, t) d x d t \\
= & -\int_{\Omega} \phi(x, 0) I_{+}^{1-\beta} y\left(x, 0^{+}\right) d x+\int_{0}^{T} \int_{\partial \Omega} y(x, t) \frac{\partial \phi(x, t)}{\partial v} d \Gamma d t \\
& -\int_{0}^{T} \int_{\partial \Omega} \frac{\partial y(x, t)}{\partial v} \phi d \Gamma d t+\int_{0}^{T} \int_{\Omega} y(x, t)\left(-D^{\beta} \phi(x, t)+\mathcal{A}(t)^{*} \phi(x, t)\right) d x d t .
\end{aligned}
$$

Lemma 3.4 We can state equations (3.1)-(3.4) in a more convenient form, first let us define the operator $\mathcal{M}$ by

$$
\begin{aligned}
& \mathcal{M} y(t)= \begin{cases}y(t-\omega), & t>\omega, \\
0, & t<\omega,\end{cases} \\
& f(t)= \begin{cases}f_{1}(t), & t>\omega, \\
D_{+}^{\beta} g+\mathcal{A}(t) g, & t<\omega,\end{cases} \\
& y(x, 0)=g(x, 0) .
\end{aligned}
$$

Then equations (3.1)-(3.4) have the following form:

$$
\begin{aligned}
& D_{+}^{\beta} y(x, t)+\mathcal{A}(t) y(x, t)+\mathcal{M} y(x, t)=f, \quad x \in \Omega, t \in(0, T), \\
& y(x, 0)=g(x, 0), \quad x \in \Omega, \\
& I_{+}^{1-\beta} y\left(x, 0^{+}\right)=y_{0}(x), \quad x \in \Omega, \\
& y(x, t)=0, \quad x \in \Gamma, t \in(0, T),
\end{aligned}
$$


We can notice that equations (3.13) and (3.1) are equivalent in $(0, T)$. But in the interval $(0, \omega)$ from equations (3.11)-(3.13) we get

$$
\begin{aligned}
& D_{+}^{\beta} y(x, t)+\mathcal{A}(t) y(x, t)=D_{+}^{\beta} g(x, t)+\mathcal{A}(t) g(x, t), \quad x \in \Omega, t \in(0, \omega), \\
& y(x, 0)=g(x, 0), \quad x \in \Omega, t=0,
\end{aligned}
$$

Lemma 3.5 By using (Theorem 3.1, Lions 1971), we can easily show that $y \equiv g$ in $(0, \omega)$. By using a constructive method we can prove the existence and uniqueness of the solution of equations (3.13)-(3.16). Firstly, we solve (3.13)-(3.16) in the subinterval $(0, \omega)$ and then in the subinterval $(\omega, 2 \omega)$ etc., until the procedure covers the whole interval $(0, T)$. In this way the solution in the previous step determines the next one. In fact, in every subinterval the solution of equations (3.13)-(3.16) exists and is unique (Theorem 3.1, Ref. [18]). If the right-hand sides of equations (3.13)-(3.16) are equal to zero, then there exists only a trivial solution of equations (3.13)-(3.16), i.e., $y \equiv 0$.

A more general case (e.g., when $\omega$ is a function of the time $t$ ) can be treated analogously as in Ref. [18] see Lemma 2.6.

Lemma 3.6 If (3.7), (3.8) holds, then the system (3.13)-(3.16) has a unique solution $y \in$ $\mathcal{W}(0, T)$.

Proof By using the coerciveness condition (3.7) and the Lax-Milgram theorem, there exists a unique solution $y(t) \in H_{0}^{1}(\Omega)$ so that

$$
\left(D_{+}^{\beta} y(t), \phi\right)_{L^{2}(Q)}+\pi(t ; y, \phi)=L(\phi) \quad \text { for all } \phi \in H_{0}^{1}(\Omega)
$$

which is equivalent to the existence of a unique solution $y(t) \in H_{0}^{1}(\Omega)$ for

$$
\left(D_{+}^{\beta} y(t), \phi\right)_{L^{2}(Q)}+((\mathcal{A}(t)+M) y(t), \phi)_{L^{2}(Q)}=L(\phi) \quad \text { for all } \phi \in H_{0}^{1}(\Omega)
$$

i.e. for

$$
\left(D_{+}^{\beta} y(t)+(\mathcal{A}(t)+M) y(t), \phi(x)\right)_{L^{2}(Q)}=L(\phi)
$$

which can be written as

$$
\int_{Q}\left(D_{+}^{\beta} y(t)+(\mathcal{A}(t)+M) y(t)\right) \phi(x) d x d t=L(\phi) \quad \text { for all } \phi \in H_{0}^{1}(\Omega) .
$$

This is known as the variational fractional Dirichlet problem, where $L(\phi)$ is a continuous linear form on $H_{0}^{1}(\Omega)$ and it takes the form

$$
L(\phi)=\int_{Q} f \phi d x d t+\int_{\Omega} y_{0} \phi(x, 0) d x, \quad f \in L^{2}(Q), y_{0} \in L^{2}(\Omega) .
$$

Then equation (3.20) is equivalent to

$$
\int_{Q}\left(D_{+}^{\beta} y(t)+\mathcal{A}(t) y(t)\right) \phi(x) d x d t=\int_{Q} f \phi d x d t+\int_{\Omega} y_{0} \phi(x, 0) d x \quad \text { for all } \phi \in H_{0}^{1}(\Omega)
$$


that is, the PDE

$$
D_{+}^{\beta} y(t)+(\mathcal{A}(t)+M) y(t)=f
$$

'tested' against $\phi(x)$.

Let us multiply both sides in (3.22) by $\phi$ and applying Green's formula (Lemma 3.2), we have

$$
\begin{aligned}
\int_{Q}\left(D_{+}^{\beta} y+(\mathcal{A}(t)+M) y\right) \phi d x d t \\
=\int_{Q} f \phi d x d t-\int_{\Omega} \phi(x, 0) I_{+}^{1-\beta} y\left(x, 0^{+}\right) d x \\
\quad+\int_{0}^{T} \int_{\partial \Omega} y \frac{\partial \phi}{\partial \nu} d \Gamma d t-\int_{0}^{T} \int_{\partial \Omega} \frac{\partial y}{\partial \nu} \phi d \Gamma d t \\
\quad+\int_{0}^{T} \int_{\Omega} y(x, t)\left(-D^{\beta} \phi(x, t)+(\mathcal{A}(t)+M)^{*} \phi(x, t)\right) d x d t=\int_{Q} f \phi d x d t
\end{aligned}
$$

whence comparing with (3.20), (3.21)

$$
\int_{\Omega} \phi(x, 0) I_{+}^{1-\beta} y\left(x, 0^{+}\right) d x-\int_{0}^{T} \int_{\partial \Omega} y \frac{\partial \phi}{\partial \nu} d \Gamma d t=\int_{\Omega} y_{0} \phi(x, 0) d x
$$

From this we deduce (3.15) and (3.16).

Lemma 3.7 We can extend our fractional problem (3.13)-(3.16) to a more general situation as follows. Let

$$
t \rightarrow \omega(t) \quad \text { be a bounded measurable function which is positive in }[0, T] \text {. }
$$

For $y \in \mathcal{W}(0, T)$, we define

$$
\mathcal{M} y(t)= \begin{cases}y(t-\omega(t)), & \text { if } t-\omega(t) \geq 0, \\ 0, & \text { if } t-\omega(t)<0 .\end{cases}
$$

We then search for $y \in \mathcal{W}(0, T)$ which is a solution of (3.13)-(3.16), $\mathcal{M}$ being given by (3.24). It may be shown (Lions, 1971) that if (3.8), (3.9) as well as (3.23) hold and if $\mathcal{M}$ is given by (3.24), then problem (3.13)-(3.16) admits a unique solution.

\section{Optimization theorem and the fractional control problem}

For a control $u \in L^{2}(Q)$ the state $y(u)$ of the system is given by

$$
\begin{aligned}
& D_{+}^{\beta} y(t ; x, u)+\mathcal{A}(t) y(t ; x, u)+\mathcal{M} y(t ; x, u)=f+u, \quad x \in \Omega, t \in(0, T), \\
& y(x, 0)=g(x, 0), \quad x \in \Omega, \\
& I_{+}^{1-\beta} y\left(x, 0^{+}\right)=y_{0}(x), \quad x \in \Omega, \\
& y(x, t)=0, \quad x \in \Gamma, t \in(0, T) .
\end{aligned}
$$


The observation equation is given by

$$
z(u)=y(u)
$$

The cost function $J(v)$ is given by

$$
J(v)=\int_{Q}\left(y(v)-z_{d}\right)^{2} d x d t+(N v, v)_{L^{2}(Q)}
$$

where $z_{d}$ is a given element in $L^{2}(\Sigma)$ and $N \in \mathcal{L}\left(L^{2}(Q), L^{2}(Q)\right)$ is a hermitian positive definite operator:

$$
(N u, u) \geq c\|u\|_{L^{2}(Q)}^{2}, \quad c>0 .
$$

Control constraints: We define $U_{a d}$ (the set of admissible controls) to be a closed, convex subset of $U=L^{2}(Q)$.

Control problem: We want to minimize $J$ over $U_{a d}$ i.e. find $u$ such that

$$
J(u)=\inf _{v \in U_{a d}} J(v)
$$

Under the given considerations we have the following theorem.

Theorem 4.1 The problem (4.8) admits a unique solution given by (4.1)-(4.4) and by

$$
\int_{Q}(p(u)+N u)(v-u) d x d t \geq 0,
$$

where $p(u)$ is the adjoint state.

Proof Since the control $u \in U_{a d}$ is optimal if and only if

$$
J^{\prime}(u)(v-u) \geq 0 \quad \text { for all } v \in U_{a d}
$$

The above condition, when explicitly calculated for this case, gives

$$
\left(y(u)-z_{d}, y(v)-y(u)\right)_{L^{2}(Q)}+(N u, v-u)_{L^{2}(Q)} \geq 0
$$

i.e.

$$
\int_{Q}\left(y(u)-z_{d}\right)(y(v)-y(u)) d x d t+(N u, v-u)_{L^{2}(Q)} \geq 0 .
$$

For the control $u \in L^{2}(Q)$ the adjoint state $p(u) \in L^{2}(Q)$ is defined by

$$
\begin{aligned}
& -D^{\beta} p(u)+\mathcal{A}^{*}(t) p(u)+\mathcal{M}^{*} p(u)=y(u)-z_{d}, \quad \text { in } Q, \\
& p(u)=0, \quad \text { on } \Sigma, \\
& p(x, T ; u)=0, \quad \text { in } \Omega .
\end{aligned}
$$


Now, multiplying equation (4.11) by $(y(v)-y(u))$ and applying Green's formula, we obtain

$$
\begin{aligned}
& \int_{Q}\left(y(u)-z_{d}\right)(y(v)-y(u)) d x d t \\
& =\int_{Q}\left(-D^{\beta} p(u)+\mathcal{A}^{*}(t) p(u)+\mathcal{M}^{*} p(u)\right)(y(v)-y(u)) d x d t \\
& =\int_{\Omega} p(x, 0) I_{+}^{1-\beta}\left(y\left(v ; x, 0^{+}\right)-y\left(u ; x, 0^{+}\right)\right) d x \\
& \quad+\int_{\Sigma} p(u)\left(\frac{\partial y(v)}{\partial v_{\mathcal{A}(t)}}-\frac{\partial y(u)}{\partial v_{\mathcal{A}(t)}}\right) d \Sigma-\int_{\Sigma} \frac{\partial p(u)}{\partial v_{\mathcal{A}(t)}}(y(v)-y(u)) d \Sigma \\
& \quad+\int_{Q} p(u)\left(D_{+}^{\beta}+\mathcal{A}(t)+\mathcal{M}\right)(y(v)-y(u)) d x d t .
\end{aligned}
$$

From (4.1), (4.4) we have

$$
\left(D_{+}^{\beta}+\mathcal{A}(t)+\mathcal{M}\right)(y(v)-y(u))=v-u,\left.\quad y(u)\right|_{\Sigma}=0,\left.\quad p(u)\right|_{\Sigma}=0 .
$$

Then we obtain

$$
\int_{Q}\left(y(u)-z_{d}\right)(y(v)-y(u)) d x d t=\int_{Q} p(u)(v-u) d x d t
$$

and hence (4.10) is equivalent to

$$
\int_{Q} p(u)(v-u) d x d t+(N u, v-u)_{L^{2}(Q)} \geq 0
$$

i.e.

$$
\int_{Q}(p(u)+N u)(v-u) d x d t \geq 0,
$$

which completes the proof.

Example 4.1 In the case of no constraints on the control $\left(\mathcal{U}_{a d}=\mathcal{U}\right)$, then (4.9) reduces to

$$
p+N u=0 \quad \text { on } \Sigma .
$$

The fractional optimal control is obtained by the simultaneous solution of the following system of fractional partial differential equations:

$$
\begin{aligned}
& D_{+}^{\beta} y+\mathcal{A}(t) y+\mathcal{M} y=f, \quad-D^{\beta} p+\mathcal{A}^{*}(t) p+\mathcal{M}^{*} p=y-z_{d} \quad \text { in } Q, \\
& y+\left.N^{-1} p\right|_{\Sigma}=0, \quad p=0 \quad \text { on } \Sigma, \\
& I_{+}^{1-\beta} y(x, 0)=y_{0}(x), \quad p(x, T)=0, \quad x \in \Omega,
\end{aligned}
$$

further

$$
u=-N^{-1}\left(\left.P\right|_{\Sigma}\right)
$$




\section{Example 4.2 Take}

$$
\mathcal{U}_{a d}=\left\{u \mid u \in L^{2}(\Sigma), u \geq 0 \text { almost everywhere on } \Sigma\right\} .
$$

The optimal control is obtained by the solution of the fractional problem

$$
\begin{aligned}
& D_{+}^{\beta} y+\mathcal{A}(t) y+\mathcal{M} y=f, \quad-D^{\beta} p+\mathcal{A}^{*}(t) p+\mathcal{M}^{*} p=y-z_{d} \quad \text { in } Q, \\
& y \geq 0, \quad p=0 \quad \text { on } \Sigma, \\
& p+N y \geq 0, \quad[p+N p]=0 \quad \text { on } \Sigma, \\
& I_{+}^{1-\beta} y(x, 0)=y_{0}(x), \quad p(x, T)=0, \quad x \in \Omega,
\end{aligned}
$$

hence

$$
u=\left.y\right|_{\Sigma}
$$

Example 4.3 We consider the fractional diffusion equation with Caputo fractional derivatives and time delay. We have

$$
\begin{aligned}
& D_{0}^{\beta} y(t)-\Delta y(t)+\mathcal{M} y=v, \quad t \in[0, T], \\
& y(0)=y_{0}, \quad x \in \Omega, \\
& y(x, t)=0, \quad x \in \Gamma, t \in(0, T),
\end{aligned}
$$

where $y_{0} \in H^{2}(\Omega) \cap H_{0}^{1}(\Omega), \Delta$ is the Laplace operator and the control $v$ belongs to $L^{2}(Q)$. We can minimize

$$
J(v)=\left\|y(v)-z_{d}\right\|_{L^{2}(Q)}^{2}+N\|v\|_{L^{2}(Q)}^{2}, \quad z_{d} \in L^{2}(Q), N>0,
$$

subject to system (4.14)-(4.16) and the optimal control $v$ will be characterized by system (4.14)-(4.16) with the adjoint system

$$
\begin{aligned}
& -D^{\beta} p(t)-\Delta p(t)+\mathcal{M}^{*} y=y-z_{d}, \quad t \in[0, T], \\
& p(x, t)=0, \quad x \in \Omega, t \in(0, T), \\
& p(x, T)=0, \quad x \in \Gamma,
\end{aligned}
$$

and with the optimality condition

$$
v=-\frac{p}{N} \quad \text { in } Q .
$$

Example 4.4 We can also consider the fractional diffusion equation with RiemannLiouville fractional derivatives and time delay:

$$
\begin{aligned}
& D_{+}^{\beta} y(t)-\Delta y(t)+\mathcal{M} y=v, \quad t \in[0, T], \\
& { }_{0} I_{T}^{1-\beta(t)} y\left(0^{+}\right)=y_{0}, \quad x \in \Omega,
\end{aligned}
$$




$$
y(x, t)=0, \quad x \in \Gamma, t \in(0, T)
$$

where ${ }_{0} I_{T}^{1-\beta(t)} y\left(0^{+}\right)=\lim _{t \rightarrow 0^{+}} I_{T}^{1-\beta(t)} y(t)$, the control $v$ belongs to $L^{2}(Q)$. We can minimize the cost function (4.17) subject to system (4.22)-(4.24) and the optimal control $v$ will be characterized by system (4.22)-(4.24) with the adjoint system (4.18)-(4.20) and with the optimality condition (4.21).

\section{Fractional Neumann problem for differential system with time delay}

Since $H_{0}^{1}(\Omega) \subset H^{1}(\Omega)$ we can show that the bilinear form (3.7) is coercive in $H^{1}(\Omega)$, that is,

$$
\pi(y, y) \geq c\|y\|_{H^{1}(\Omega)}^{2}, \quad c>0 \text { for all } y \in H^{1}(\Omega)
$$

Also we have the following.

Lemma 5.1 If (5.1) holds, then there exists a unique solution $y \in H^{1}(\Omega)$ such that

$$
\begin{aligned}
& D_{+}^{\beta} y+\mathcal{A}(t) y+\mathcal{M} y=f \quad \text { in } Q, \\
& \frac{\partial y}{\partial \nu_{\mathcal{A}(t)}}=h \quad \text { on } \Sigma, \\
& I_{+}^{1-\beta} y\left(0^{+}\right)=y_{0}(x), \quad x \in \Omega,
\end{aligned}
$$

where

$$
\frac{\partial y}{\partial \nu_{\mathcal{A}(t)}}=\sum_{i, j=1}^{n} a_{i j} \frac{\partial y}{\partial x_{j}} \cos \left(n, x_{j}\right) \text { on } \Gamma,
$$

$\cos \left(n, x_{j}\right)$ is the ith direction cosine of $n, n$ being the normal at $\Gamma$ exterior to $\Omega$.

Proof By using the coerciveness condition (5.1) and the Lax-Milgram lemma, there exists a unique solution $y \in H^{1}(\Omega)$ so that

$$
\int_{Q} y\left(-D^{\beta} \psi+\mathcal{A}^{*} \psi+\mathcal{M}^{*} \psi\right) d x d t=M(\psi) \text { for all } \psi \in H^{1}(\Omega) .
$$

This is known as the fractional Neumann problem, where $M(\psi)$ is a continuous linear form on $H^{1}(\Omega)$ taking the form

$$
\begin{aligned}
& M(\psi)=\int_{Q} f \psi d x d t+\int_{\Omega} y_{0} \psi(x, 0) d x-\int_{\Sigma} h \frac{\partial \psi}{\partial \nu_{A^{*}}} d \Sigma, \\
& f \in L^{2}(Q), y_{0} \in L^{2}(\Omega), h \in H^{1}(\Sigma) .
\end{aligned}
$$

Equation (5.5) is equivalent to

$$
D_{+}^{\beta} y+\mathcal{A}(t) y+\mathcal{M} y=f \quad \text { on } Q .
$$


Let us multiply both sides in (5.7) by $\psi$ and by using Green's formula, we get

$$
\begin{aligned}
\int_{Q}\left(D_{+}^{\beta} y+\mathcal{A}(t) y+\mathcal{M} y\right) \psi d x d t \\
=\int_{Q} f \psi d x d t-\int_{\Omega} \psi(x, 0) I_{+}^{1-\beta} y\left(x, 0^{+}\right) d x \\
\quad+\int_{0}^{T} \int_{\partial \Omega} y \frac{\partial \psi}{\partial \nu} d \Gamma d t-\int_{0}^{T} \int_{\partial \Omega} \frac{\partial y}{\partial \nu} \psi d \Gamma d t \\
\quad+\int_{0}^{T} \int_{\Omega} y(x, t)\left(-D^{\beta} \psi(x, t)+\mathcal{A}^{*}(t) \psi(x, t)+\mathcal{M}^{*} \psi(x, t)\right) d x d t=\int_{Q} f \psi d x d t,
\end{aligned}
$$

whence comparing with (5.5), (5.6)

$$
\int_{\Omega} \psi(x, 0) I_{+}^{1-\beta} y\left(x, 0^{+}\right) d x+\int_{0}^{T} \int_{\partial \Omega} \psi \frac{\partial y}{\partial \nu} d \Gamma d t=\int_{\Omega} y_{0} \psi(x, 0) d x+\int_{0}^{T} \int_{\partial \Omega} h \psi d \Gamma d t .
$$

From this we deduce (5.3) and (5.4).

\section{Fractional minimization theorem and boundary control problem}

We consider the space $U=L^{2}(\Sigma)$ (the space of controls), for every control $u \in U$, the state of the system $y(u) \in H^{1}(\Omega)$ is given by the solution of

$$
\begin{aligned}
& D_{+}^{\beta} y(u)+\mathcal{A}(t) y(u)+\mathcal{M} y(u)=f \quad \text { in } Q, \\
& \frac{\partial y(u)}{\partial \nu_{\mathcal{A}(t)}}=u \quad \text { on } \Sigma, \\
& I_{+}^{1-\beta} y(x, 0 ; u)=y_{0}(x), \quad x \in \Omega .
\end{aligned}
$$

For the observation, we consider the following two cases:

(i)

$$
z(u)=y(u)
$$

(ii) Observation of the final state

$$
z(u)=y(x, T ; u) .
$$

Case (i): The cost function is given by

$$
J(v)=\int_{Q}\left(y(v)-z_{d}\right)^{2} d x d t+(N v, v)_{L^{2}(\Sigma)}, \quad z_{d} \in L^{2}(Q)
$$

where $N \in \mathcal{L}\left(L^{2}(\Sigma), L^{2}(\Sigma)\right), N$ is hermitian positive definite:

$$
(N u, u)_{L^{2}(\Sigma)} \geq c\|u\|_{L^{2}(\Sigma)}^{2}, \quad c>0 .
$$

Control constraints: We define $U_{a d}$ (set of admissible controls) to be a closed, convex subset of $U=L^{2}(\Sigma)$. 
Control problem: We wish to find

$$
\inf _{v \in U_{a d}} J(v)
$$

Under the given considerations we have the following theorem.

Theorem 6.1 Assume that (6.7) holds and the cost function is given by (6.6). The optimal control $u$ is characterized by (6.1), (6.2), (6.3) together with

$$
\begin{aligned}
& -D^{\beta} p(u)+\mathcal{A}^{*}(t) p(u)+\mathcal{M}^{*} p(u)=y(u)-z_{d} \quad \text { in } Q \\
& \frac{\partial p(u)}{\partial \nu_{\mathcal{A}^{*}}}=0 \quad \text { on } \Sigma \\
& p(x, T ; u)=0, \quad x \in \Omega,
\end{aligned}
$$

and the optimality condition is

$$
\int_{\Sigma}(p(u)+N u)(v-u) d \Sigma \geq 0 \quad \forall v \in U_{a d}
$$

where $p(u)$ is the adjoint state.

Proof The control $u \in U_{a d}$ is optimal if and only if

$$
J^{\prime}(u)(v-u) \geq 0 \quad \forall v \in U_{a d}
$$

that is,

$$
\left(y(u)-z_{d}, y(v)-y(u)\right)_{L^{2}(Q)}+(N u, v-u)_{U} \geq 0
$$

The adjoint state is given by the solution of the adjoint Neumann problem (6.9), (6.10), (6.11). Multiplying equation (6.9) by $y(v)-y(u)$ and by using Green's formula, having in mind the conditions in (6.1), (6.2), we obtain

$$
\begin{aligned}
\int_{Q} & \left(y(u)-z_{d}\right)(y(v)-y(u)) d x d t \\
= & \int_{Q}\left(-D^{\beta} p(u)+\mathcal{A}^{*}(t) p(u)+\mathcal{M}^{*} p(u)\right)(y(v)-y(u)) d x d t \\
= & -\int_{\Omega} p(x, 0) I_{+}^{1-\beta}\left(y\left(v ; x, 0^{+}\right)-y\left(u ; x, 0^{+}\right)\right) d x \\
& +\int_{\Sigma} p(u)\left(\frac{\partial}{\partial v_{\mathcal{A}(t)}} y(v)-\frac{\partial}{\partial v_{\mathcal{A}(t)}} y(u)\right) d \Sigma \\
& \quad-\int_{\Sigma} \frac{\partial}{\partial v_{\mathcal{A}}^{*}} p(u)(y(v)-y(u)) d \Sigma+\int_{Q} p(u)\left(D_{+}^{\beta}+\mathcal{A}(t)+\mathcal{M}\right)(y(v)-y(u)) d x d t \\
= & \int_{\Sigma} p(u)(v-u) d \Sigma
\end{aligned}
$$


Hence we substituted from (6.15) in (6.14), to get

$$
\int_{\Sigma} p(u)(v-u) d \Sigma+(N u, v-u)_{L^{2}(\Sigma)} \geq 0
$$

i.e.

$$
\int_{\Sigma}(p(u)+N u)(v-u) d \Sigma \geq 0 \quad \forall v \in U_{a d}
$$

which completes the proof.

Example 6.1 In the case of no constraints on the control $\left(\mathcal{U}_{a d}=\mathcal{U}\right)$, then (6.12) reduces to

$$
p+N u=0 \quad \text { on } \Sigma \text {. }
$$

The fractional optimal control is obtained by the simultaneous solution of the following system of fractional partial differential equations:

$$
\begin{aligned}
& D_{+}^{\beta} y+\mathcal{A}(t) y+\mathcal{M} y=f, \quad-D^{\beta} p+\mathcal{A}^{*}(t) p+\mathcal{M}^{*} p=y-z_{d} \quad \text { in } Q, \\
& \left.\frac{\partial y}{\partial \nu_{\mathcal{A}}}\right|_{\Sigma}+\left.N^{-1} p\right|_{\Sigma}=0, \quad \frac{\partial p}{\partial v_{A^{*}}}=0 \quad \text { on } \Sigma, \\
& I_{+}^{1-\beta} y(x, 0)=y_{0}(x), \quad p(x, T)=0, \quad x \in \Omega,
\end{aligned}
$$

further

$$
u=-N^{-1}\left(\left.P\right|_{\Sigma}\right)
$$

\section{Example 6.2 Take}

$\mathcal{U}_{a d}=\left\{u \mid u \in L^{2}(\Sigma), u \geq 0\right.$ almost everywhere on $\left.\Sigma\right\}$.

The optimal control is obtained by the solution of the fractional problem

$$
\begin{aligned}
& D_{+}^{\beta} y+\mathcal{A}(t) y+\mathcal{M} y=f, \quad-D^{\beta} p+\mathcal{A}^{*}(t) p+\mathcal{M}^{*} p=y-z_{d} \quad \text { in } Q, \\
& \frac{\partial y}{\partial \nu_{\mathcal{A}}} \geq 0, \quad \frac{\partial p}{\partial \nu_{\mathcal{A}^{*}}}=0 \quad \text { on } \Sigma, \\
& p+N \frac{\partial y}{\partial \nu_{\mathcal{A}}} \geq 0, \quad \frac{\partial y}{\partial \nu_{\mathcal{A}}}\left[p+N \frac{\partial y}{\partial \nu_{\mathcal{A}}}\right]=0 \quad \text { on } \Sigma, \\
& I_{+}^{1-\beta} y(x, 0)=y_{0}(x), \quad p(x, T)=0, \quad x \in \Omega,
\end{aligned}
$$

hence

$$
u=\left.\frac{\partial y}{\partial v_{\mathcal{A}}}\right|_{\Sigma}
$$


Case (ii): Observation of the final state

$$
z(u)=y(x, T ; u)
$$

The cost function is given by

$$
J(v)=\int_{\Omega}\left(y(x, T ; v)-z_{d}\right)^{2} d x+(N v, v)_{L^{2}(\Sigma)}, \quad z_{d} \in L^{2}(\Omega) .
$$

The adjoint state is defined by

$$
\begin{aligned}
& -D^{\beta} p(u)+\mathcal{A}^{*}(t) p(u)+\mathcal{M}^{*} p(u)=0 \quad \text { in } Q, \\
& \frac{\partial p(u)}{\partial v_{\mathcal{A}^{*}}}=0, \quad \text { on } \Sigma, \\
& p(x, T ; u)=y(x, T ; u)-z_{d}(x), \quad x \in \Omega,
\end{aligned}
$$

and the optimality condition is

$$
\int_{\Sigma}(p+N u)(v-u) d \Sigma \geq 0 \quad \forall v \in U_{a d}
$$

where $p(u)$ is the adjoint state.

Example 6.3 In the case of no constraints on the control $\left(\mathcal{U}_{\text {ad }}=\mathcal{U}\right)$, then $(6.16)$ reduces to

$$
p+N u=0 \quad \text { on } \Sigma \text {. }
$$

The optimal control is obtained by the simultaneous solution of the following system of fractional partial differential equations:

$$
\begin{aligned}
& D_{+}^{\beta} y+\mathcal{A}(t) y+\mathcal{M} y=f, \quad-D^{\beta} p+\mathcal{A}^{*}(t) p+\mathcal{M}^{*} p=0 \quad \text { in } Q, \\
& \left.\frac{\partial y}{\partial \nu_{\mathcal{A}}}\right|_{\Sigma}+\left.N^{-1} p\right|_{\Sigma}=0, \quad \frac{\partial p}{\partial \nu_{\mathcal{A}}^{*}}=0 \quad \text { on } \Sigma, \\
& I_{+}^{1-\beta} y(x, 0)=y_{0}(x), \quad p(x, T)=y(x, T ; u)-z_{d}(x), \quad x \in \Omega,
\end{aligned}
$$

further

$$
u=-N^{-1}\left(\left.P\right|_{\Sigma}\right)
$$

Example 6.4 If we take

$$
\mathcal{U}_{a d}=\left\{u \mid u \in L^{2}(\Sigma), u \geq 0 \text { almost everywhere on } \Sigma\right\},
$$

then (6.16) is equivalent to

$$
u \geq 0, \quad p(u)+N u \geq 0, \quad u(p(u)+N u)=0 \quad \text { on } \Sigma .
$$


Example 6.5 We consider the Neumann fractional diffusion equation with Caputo fractional derivatives and time delay,

$$
\begin{aligned}
& D_{0}^{\beta} y(t)-\Delta y(t)+\mathcal{M} y=v, \quad t \in[0, T], \\
& y(0)=y_{0}, \quad x \in \Omega, \\
& \frac{\partial y(x, t)}{\partial \nu_{\mathcal{A}}}=0, \quad x \in \Gamma, t \in(0, T),
\end{aligned}
$$

where $y_{0} \in H^{2}(\Omega) \cap H_{0}^{1}(\Omega), \Delta$ is the Laplace operator and the control $v$ belongs to $L^{2}(Q)$. We can minimize

$$
J(v)=\left\|y(v)-z_{d}\right\|_{L^{2}(Q)}^{2}+N\|v\|_{L^{2}(Q)}^{2}, \quad z_{d} \in L^{2}(Q), N>0,
$$

subject to system (6.17)-(6.19) and the optimal control $v$ will be characterized by system (6.17)-(6.19) with the adjoint system

$$
\begin{aligned}
& -D^{\beta} p(t)-\Delta p(t)+\mathcal{M}^{*} y=y-z_{d}, \quad t \in[0, T], \\
& \frac{\partial p(x, t)}{\partial \nu_{\mathcal{A}^{*}}}=0, \quad x \in \Omega, t \in(0, T), \\
& p(x, T)=0, \quad x \in \Gamma,
\end{aligned}
$$

and with the optimality condition

$$
v=-\frac{p}{N} \quad \text { in } Q .
$$

Example 6.6 We can also consider the Neumann fractional diffusion equation with Riemann-Liouville fractional derivatives and time delay:

$$
\begin{aligned}
& D_{+}^{\beta} y(t)-\Delta y(t)+\mathcal{M} y=v, \quad t \in[0, T], \\
& { }_{0} I_{T}^{1-\beta(t)} y\left(0^{+}\right)=y_{0}, \quad x \in \Omega, \\
& \frac{\partial y(x, t)}{\partial \nu_{\mathcal{A}}}=0, \quad x \in \Gamma, t \in(0, T),
\end{aligned}
$$

where ${ }_{0} I_{T}^{1-\beta(t)} y\left(0^{+}\right)=\lim _{t \rightarrow 0^{+}}{ }_{0} I_{T}^{1-\beta(t)} y(t)$, the control $v$ belongs to $L^{2}(Q)$. We can minimize the cost function (4.17) subject to system (6.25)-(6.27) and the optimal control $v$ will be characterized by system (6.25)-(6.27) with the adjoint system (6.21)-(6.23) and with the optimality condition (6.24).

Remark 6.2 If we take $\beta=1$ in the previous sections we obtain the classical results in the optimal control with integer derivatives.

\section{Conclusions}

An analytical scheme for fractional optimal control of differential systems with time delay is considered. The fractional derivatives were defined in the Riemann-Liouville sense. The analytical results were given in terms of Euler-Lagrange equations for the fractional 
optimal control problems. The formulation presented and the resulting equations are very similar to those for classical optimal control problems. The optimization problem presented in this paper constitutes a generalization of the optimal control problem of parabolic systems with Dirichlet and Neumann boundary conditions, considered by Lions et al. [21, 22], Podlubny [27], Oldham et al. [27], Bahaa et al. [1-4, 13-15] and Kotarski et al. $[23,28-30]$, to the fractional optimal control problem for second order systems with time delay.

\section{Competing interests}

The author declares that he has no competing interests.

\section{Acknowledgements}

The author would like to express his gratitude to the anonymous reviewers for their very valuable remarks and comments.

Received: 30 September 2016 Accepted: 21 February 2017 Published online: 01 March 2017

\section{References}

1. Bahaa, GM: Optimality conditions for cooperative parabolic systems governed by Schrödinger operator with control constraints. Asian-Eur. J. Math. 1, 131-146 (2008)

2. Bahaa, GM: Optimality conditions for infinite order distributed parabolic systems with multiple time delays given in integral form. J. Appl. Math. 2012, 672947 (2012)

3. Bahaa, GM, Kotarski, W: Time-optimal control of infinite order distributed parabolic systems involving multiple time-varying lags. Numer. Funct. Anal. Optim. 37(9), 1066-1088 (2016)

4. Bahaa, GM, Tharwat, MM: Optimal boundary control for infinite variables parabolic systems with time lags given in integral form. Iran. J. Sci. Technol. 3, 277-291 (2012)

5. Lions, JL: Optimal Control of Systems Governed by Partial Differential Equations, Band 170. Springer, Berlin (1971)

6. Lions, JL, Magenes, E: Non-Homogeneous Boundary Value Problem and Applications, vol. I. Springer, New York (1972)

7. Kotarski, W, El-Saify, HA, Bahaa, GM: Optimal control of parabolic equation with an infinite number of variables for non-standard functional and time delay. IMA J. Math. Control Inf. 19, 461-476 (2002)

8. Agrawal, OP: Formulation of Euler-Lagrange equations for fractional variational problems. J. Math. Anal. Appl. 272, 368-379 (2002)

9. Agrawal, OP: A general formulation and solution scheme for fractional optimal control problems. Nonlinear Dyn. 38, 323-337 (2004)

10. Agrawal, OP: Fractional optimal control of a distributed system using eigenfunctions. J. Comput. Nonlinear Dyn. 3(2), $1-6(2008)$

11. Agrawal, OP, Baleanu, D: A Hamiltonian formulation and direct numerical scheme for fractional optimal control problems. J. Vib. Control 13(9-10), 1269-1281 (2007)

12. Agrawal, OP, Defterli, O, Baleanu, D: Fractional optimal control problems with several state and control variables J. Vib. Control 16(13), 1967-1976 (2010)

13. Bahaa, GM: Fractional optimal control problem for variational inequalities with control constraints. IMA J. Math Control Inf. 6(33), 1-16 (2016)

14. Bahaa, GM: Fractional optimal control problem for differential system with control constraints. Filomat 30(8), 2177-2189 (2016)

15. Bahaa, GM: Fractional optimal control problem for infinite order system with control constraints. Adv. Differ. Equ. 2016, 250 (2016)

16. Baleanu, D, Muslih, SI: Lagrangian formulation on classical fields within Riemann-Liouville fractional derivatives. Phys. Scr. 72(2-3), 119-121 (2005)

17. Baleanu, D, Avkar, T: Lagrangian with linear velocities within Riemann-Liouville fractional derivatives. Nuovo Cimento B 119, 73-79 (2004)

18. Baleanu, DA, Agrawal, OP: Fractional Hamilton formalism within Caputo's derivative. Czechoslov. J. Phys. 56(10/11), 1087-1092 (2006)

19. Baleanu, D, Defterli, O, Agrawal, OP: Central difference numerical scheme for fractional optimal control problems. J. Vib. Control 15(4), 583-597 (2009)

20. Frederico Gastao, F, Torres Delfim, FM: Fractional optimal control in the sense of Caputo and the fractional Noether's theorem. Int. Math. Forum 3(10), 479-493 (2008)

21. Jajarmi, A, Baleanu, D: Suboptimal control of fractional-order dynamic systems with delay argument. J. Vib. Control (2017). doi:10.1177/1077546316687936

22. Jarad, F, Maraba, T, Baleanu, D: Fractional variational optimal control problems with delayed arguments. Nonlinear Dyn. 62, 609-614 (2010)

23. Jarad, F, Maraba, T, Baleanu, D: Higher order fractional variational optimal control problems with delayed arguments. Appl. Math. Comput. 218, 9234-9240 (2012)

24. Mophou, GM: Optimal control of fractional diffusion equation. Comput. Math. Appl. 61, 68-78 (2011)

25. Mophou, GM: Optimal control of fractional diffusion equation with state constraints. Comput. Math. Appl. 62, 1413-1426 (2011)

26. Mophou, GM, Fotsing, JM: Optimal control of a fractional diffusion equation with delay. J. Adv. Math. 6(3), 1017-1037 (2014)

27. Oldham, KB, Spanier, J: The Fractional Calculus. Academic Press, New York (1974) 
28. Defterli, O, D'Elia, M, Du, Q, Gunzburger, M, Lehoucq, R, Meerschaert, MM: Fractional diffusion on bounded domains. Fract. Calc. Appl. Anal. 18(2), 342-360 (2015)

29. Doha, EH, Bhrawy, AH, Baleanu, D, Ezz-Eldien, SS, Hafez, RM: An efficient numerical scheme based on the shifted orthonormal Jacobi polynomials for solving fractional optimal control problems. Adv. Differ. Equ. 2015, 15 (2015)

30. Podlubny, I: Fractional Differential Equations. Academic Press, San Diego (1999)

Submit your manuscript to a SpringerOpen ${ }^{\odot}$ journal and benefit from:

$\rightarrow$ Convenient online submission

Rigorous peer review

- Immediate publication on acceptance

- Open access: articles freely available online

- High visibility within the field

- Retaining the copyright to your article

Submit your next manuscript at $>$ springeropen.com 